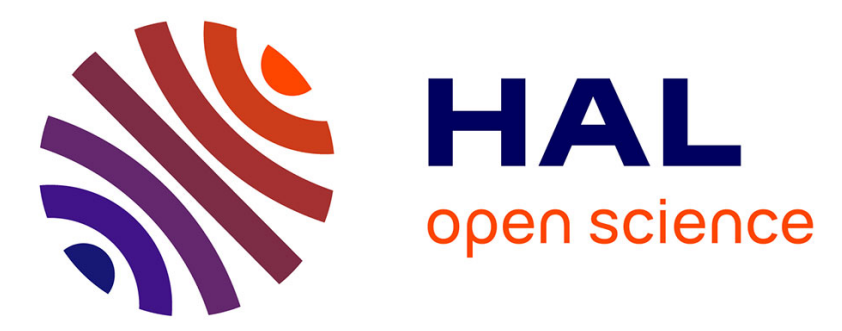

\title{
Quantitative temperature field measurements on a non-gray multi-materials scene by thermoreflectometry
}

Rémi Gilblas, Thierry Sentenac, D. Hernandez, Yannick Le Maoult

\section{To cite this version:}

Rémi Gilblas, Thierry Sentenac, D. Hernandez, Yannick Le Maoult. Quantitative temperature field measurements on a non-gray multi-materials scene by thermoreflectometry. Infrared Physics and Technology, 2014, 66, p. 70-77. 10.1016/j.infrared.2014.05.014 . hal-01611999

\author{
HAL Id: hal-01611999 \\ https://hal.science/hal-01611999
}

Submitted on $10 \mathrm{Jul} 2018$

HAL is a multi-disciplinary open access archive for the deposit and dissemination of scientific research documents, whether they are published or not. The documents may come from teaching and research institutions in France or abroad, or from public or private research centers.
L'archive ouverte pluridisciplinaire $\mathbf{H A L}$, est destinée au dépôt et à la diffusion de documents scientifiques de niveau recherche, publiés ou non, émanant des établissements d'enseignement et de recherche français ou étrangers, des laboratoires publics ou privés. 


\title{
Quantitative temperature field measurements on a non-gray multi-materials scene by thermoreflectometry
}

\author{
R. Gilblas ${ }^{*, 1}$, T. Sentenac ${ }^{1}$, D. Hernandez ${ }^{2}$, Y. Le Maoult ${ }^{1}$ \\ Toulouse University, Mines Albi, ICA (Institut Clement Ader), Campus Jarlard, F-81013 Albi cedex 09, France \\ CNRS, B.P. 5, 66125 Font-Romeu Cedex, France
}

\section{H I G H L I G H T S}

- We evaluate experimentally a non-contact camera based temperature measurement method called thermoreflectometry. - This method is based on a continuous evaluation of emissivity by reflectometry.

- A heterogeneous multi-materials scene composed of dielectrics and metals is elaborated and characterized by spectrometry.

- True temperature fields are retrieved on this complex scene and compared to reference methods.

\section{A B S T R A C T}

This article addresses the problem of measuring an accurate temperature field on a multi-materials scene composed of two dielectric and one metallic materials. The measurements of thermo-radiative properties demonstrate that the scene exhibit very different emissivity spectra and thermal conductivities inducing high thermal gradients. From these radiative properties, the calculation of the theoretical temperature error of conventional passive methods highlights that a method may be suitable for measuring only one material but no method provides satisfactory measurements of the sets of materials. The proposed method, called thermoreflectometry, performs a simultaneous measurement on all materials thanks to an on-line indirect determination of emissivity based on a bidirectional reflectivity measurement. Its temperature error is compared to that of the selected passive method for each material through an experimental validation on the multi-materials scene. These results highlight the accuracy of thermoreflectometry and shows opening prospects for the on-line temperature control of dynamical processes.

Camera

\section{Introduction}

For numerous scientific or industrial projects in aeronautical [1], nuclear [2] or space [3] domains, thermal diagnosis is a major issue. The measured hot surfaces under process are often composed of different opaque materials, metallic or dielectric, and are submitted to spatially variable high heat flux. These surfaces also exhibit some strong surface-state variations (localized oxidations, delaminations) and some possibly steep temperature gradients (convection effects, localized heating, multimaterial objects).

* Corresponding author at: Toulouse University, Mines Albi, ICA (Institut Clement Ader), Campus Jarlard, F-81013 Albi cedex 09, France. Tel.: +33 563493198; fax: +33 563493242.

E-mail address: rgilblas@mines-albi.fr (R. Gilblas).

URL: http://www.institut-clement-ader.org (R. Gilblas).

1 ICA (Institut Clement Ader).

2 Processes, Materials and Solar Energy (PROMES).
In our laboratory, a multi-material scene representing such measurement case has been developed and is displayed in Fig. 1. The scene exhibits different materials (metallic and dielectric), with different emissivity spectra (increasing and decreasing with wavelength) and thermal conductivities (low and high), and these properties may vary with time. This scene regroups all the major problems of a thermal diagnosis based on temperature measurements with non-contact and non-invasive methods. These methods involve an analysis of the flux emitted by the scene. Unfortunately, this flux is a complex function of mainly two parameters: temperature and emissivity. The major problem for non-invasive methods is then to separate in the signal obtained $S^{r}$ the temperature and emissivity influence, as shown by Eq. (1).

$S^{r}(\lambda, T)=\varepsilon^{r}(\lambda, T) S_{0}(\lambda, T)$

For a scene involved in dynamical processes, the emissivity is always unknown, but, in addition, varies during the measurement with temperature and surface's roughness. To provide a 


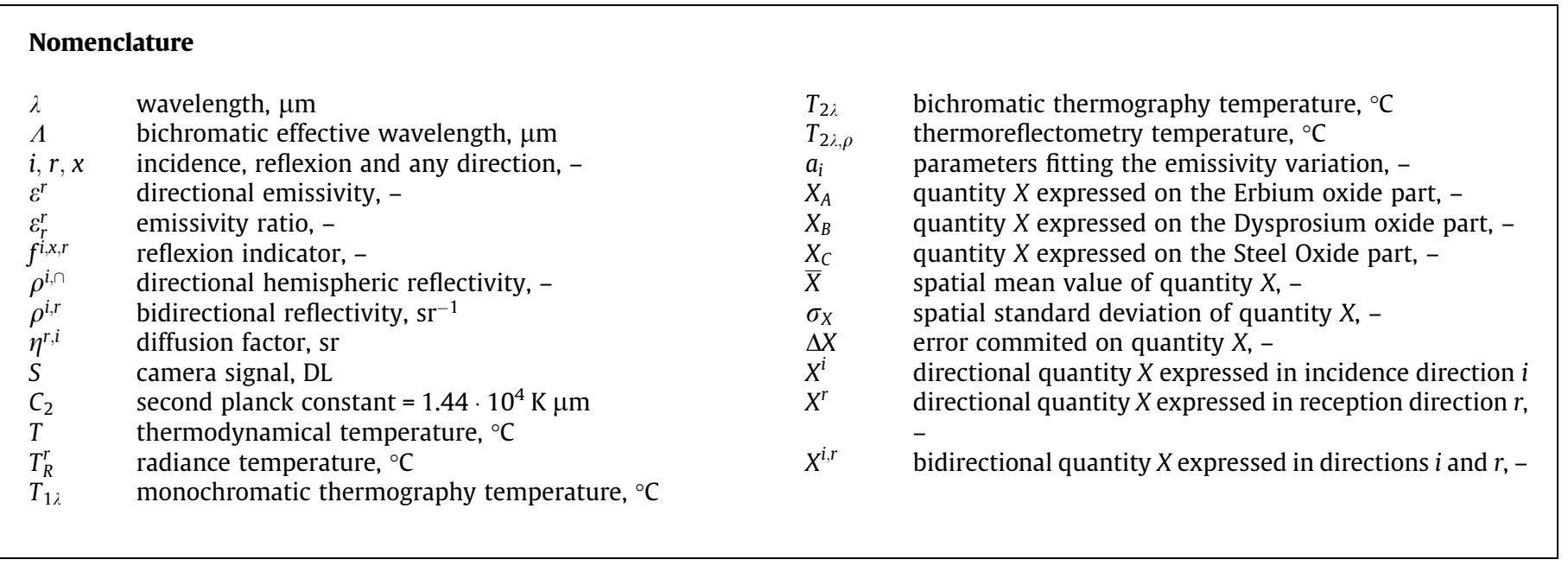

quantitative temperature measurement, non-invasive techniques have to take into account these emissivity variations which are specific to each material of the measured scene. This article then addresses the problem of an accurate, in situ and on-line measurement of the true temperature field on a multi-material scene under real dynamical conditions with both emissivity and temperature gradients.

The non-intrusive methods based on the definition of emissivity [4] (comparison of the object's radiance to the black body's radiance at the same temperature) are not considered here because they are not suitable for dynamic processes. However, for dielectric materials, monochromatic thermography at Christiansen's wavelength (around $10 \mu \mathrm{m}$, see[5]), where emissivity is equal to unity, can be an elegant approach. Performing thermography at these wavelengths is possible with a specific filter for each material. Although the Christiansen's wavelength depends slightly on temperature and surface, the filter would be effective on a restricted temperature range and a relatively homogeneous surface to minimize the uncertainty of temperature measurement. For metallic materials, bichromatic thermography [6-8], can perform a real time temperature field measurement. Bichromatic thermography assumes that the emissivity ratio at two wavelengths is known and constant. Unfortunately, emissivity ratio of metallic material usually decreases with temperature and oxidations. Multiwavelength thermography [9] then infers that the emissivity variation versus wavelength follows a particular law (linear, polynomial or exponential). For a multi-materials scene with dielectric and metallic materials, designing a law suitable for all variations of emissivity materials can be tricky. These passive methods are suit-

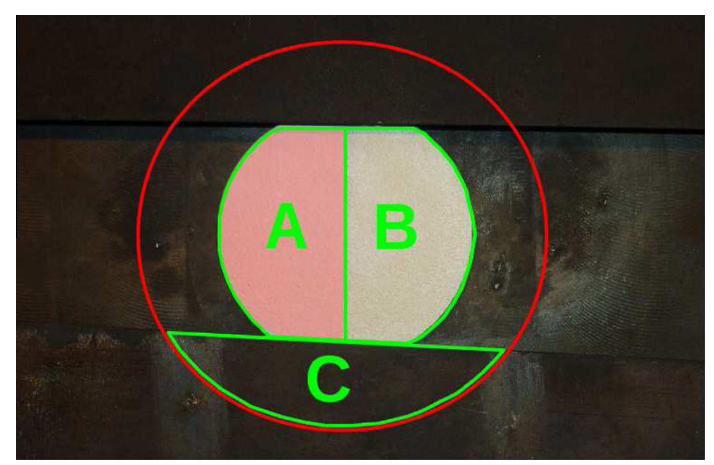

Fig. 1. Photograph of the complex scene mounted on the heating element. Item $A$ : Erbium oxide part $\left(\mathrm{ZrO}_{2}+\mathrm{Er}_{2} \mathrm{O}_{3}\right)$. Item B: Dysprosium oxide part $\left(\mathrm{ZrO}_{2}+\mathrm{Dy}_{2} \mathrm{O}_{3}\right)$. Item C: Steel Oxide flange. able for temperature field measurement on some specific materials but their application to a multi-materials scene is difficult. In addition, they are often very limited for dynamical conditions, when in situ properties quickly go off initial hypothesis. Without making assumptions on the emissivity behavior, the best approach would be to perform an emissivity measurement online and in situ with an active thermography method which require an external excitation of the material. This excitation creates a heat increase on the material surface, which is detected by the camera. This excitation can be optical (lock-in [10], pulsed or stimulated thermography [11]), mechanical (vibrothermography [12]), acoustic (thermosonics [13]), or magnetic (Eddy-current thermography [14]). These techniques all exhibit the same drawback: the difficulty of extrapolating the excitation source size to a surface-like measurement. Thanks to Kirchhoff's laws, active methods enabling the determination of emissivity via a reflection factor require less energy. Methods involving reflectance measurement are usually realized by an integrating sphere [15]. Flash assisted multiwavelength pyrometry (FAMP) [16] is more complete and take into account external radiations. Unfortunately, these methods require laboratory equipment (integrating sphere, parabolic mirrors, a spectrometer and an in situ reference) with a low ability to be integrated into embedded systems. On the other hand, pyroreflectometry or thermoreflectometry $[17,18]$ performs an on-line evaluation of the reflection factor with only two low-power lasers. For opaque materials, the true temperature field measurement is computed from a measurement of bidirectional reflectivity and radiance temperature achieved with a specific near infrared radiometric model [19]. An extensive sensitivity simulation study of this method is provided in [20], and leads to the dimensioning of a prototype.

The novelty aspect presented in this paper is the challenging case of retrieving an accurate temperature field on a scene composed of different materials (dielectric and metallic) heated to different temperatures. After a description of radiative properties of the multi-materials scene, the conventional passive methods, such as monochromatic, bichromatic and multiwavelengths thermography, are evaluated in a simulation study. This comparison is also continuing in the experimental section. Christiansen's monochromatic thermography is carried out on the dielectric materials, and bichromatic thermography on the oxide metal part, and these temperatures constitute the reference temperature for each part. The errors of thermoreflectometry are then calculated and used to evaluate the accuracy of the method. This method also provides the emissivity field of the scene, which is a relevant parameter to evaluate the physical consistency of the overall results. 
The paper is organized as follows. In Section 2, the thermoradiative properties of the multi-materials scene are measured. From these values, the theoretical temperature error of passive methods (monochromatic, bichromatic and multiwavelength thermography), and their accuracy are calculated. In Section 3, theoretical basis, apparatus and influencing quantities are outlined. Finally, temperature field measurements are carried out on the multimaterials scene by thermoreflectometry in Section 4 and the results are compared to monochromatic and bichromatic thermography. The main benefits of thermoreflectometry compared to passive methods are then discussed.

\section{Multi-materials scene and theoretical temperature error of passive methods}

This section describes the multi-materials scene composed of metallic and dielectric materials in terms of thermo-radiative properties measurement (emissivity and thermal conductivity). The theoretical temperature errors of passive methods (monochromatic, bichromatic and multiwavelength thermography) are estimated on this multi-materials scene with a high thermal and emissivity gradient.

\subsection{Multi-materials scene}

The scene suggested is a disk $3 \mathrm{~cm}$ in diameter and $3 \mathrm{~mm}$ thick, stuck between two Steel Oxide flanges, presented in Fig. 1. It is composed of three parts: the left part (item A), slightly pink, is a Zirconia $\left(\mathrm{ZrO}_{2}\right)$ sample doped at $30 \%$ in mass with Erbium oxide $\left(\mathrm{Er}_{2} \mathrm{O}_{3}\right)$. The right part (item $\left.\mathrm{B}\right)$, white, is a Zirconia sample doped at $30 \%$ with Dysprosium oxide $\left(\mathrm{Dy}_{2} \mathrm{O}_{3}\right)$. These two parts were prepared by mixing powders of both materials (Zirconia and dopants) and then sintering. The below part is one of the two flanges, composed of Steel Oxide.

\subsection{Thermo-radiative properties}

The measurements of emissivities and thermal conductivities are provided in this section.

\subsubsection{Emissivity determination}

The emissivity determination is divided in two items: the first one, at room temperature, investigates the radiative properties for near infrared (NIR) wavelengths, where thermoreflectometry operates, and where the sensibility for high temperatures is maximum. Secondly, the radiative properties for temperatures from 500 to $1000{ }^{\circ} \mathrm{C}$ in the middle infrared (MIR) are measured, to locate the Christiansen's wavelength.

For the NIR measurements, a Bruker Infrared Fourier Transform spectrometer equipped with an Infragold integrating sphere enables the measurement of reflectance. Following Kichhoffs' law, for an opaque material, the sum of absorptivity and hemispherical reflectance is equal to unity. Then, at thermal equilibrium, absorptivity is equal to emissivity, so the sum of emissivity and reflectance is equal to 1 .

As shown in the (a) part of Fig. 2, the emissivity of dielectrics vary a lot with wavelength due to absorption bands. Moreover, for some spectral bands, their variations are inverted. On the other hand, for the Steel Oxide part, emissivity is high and varies little with wavelength. This small spectral decrease of emissivity is typical for metallic oxides. For this part of the sample, and as the gray body hypothesis is valid, bichromatic thermography should be applied.

For the MIR measurements, the CEMHTI laboratory performs a direct calculation of the emissivity by a comparison with a blackbody only on dielectric materials. The part (b) of the spectrum presented Fig. 2, shows strong similarities for the two dielectrics. Christiansen's wavelength is equal to $12.8 \mu \mathrm{m}$, it matches with an emissivity equal to one and does almost not vary with temperature. As characterized in reference [21], this wavelength is the same as the Christiansen's wavelength of Zirconia. Radiative properties in the MIR are then governed by pure Zirconia, and dopants have little impact. This Christiansen's wavelength will be used in Section 4 to provide a reference temperature on the dielectric parts of the scene.

\subsubsection{Thermal conductivity measurement}

The thermal conductivity $\lambda_{c}$ is measured with the method of the hot disk [22]. It consists in inserting a heating probe between two identical pieces of the sample under study, and to measure the temperature variation, which is linked with the thermal conductivity, given with $5 \%$ accuracy. The values found are $0.96 \mathrm{~W} \mathrm{~m}^{-1} \mathrm{~K}^{-1}$ for the Erbium oxide part and $0.73 \mathrm{~W} \mathrm{~m}^{-1} \mathrm{~K}^{-1}$ for the Dysprosium oxide part, which are close to pure Zirconia values (typically between 1 and $2 \mathrm{~W} \mathrm{~m}^{-1} \mathrm{~K}^{-1}$ [23]). The resolution of the 1-D Fourier equation with the assumption of same radiative and convection losses on this sample gives a surface temperature difference between the two parts of $34{ }^{\circ} \mathrm{C}$ for a $1000^{\circ} \mathrm{C}$ backside uniform heating.
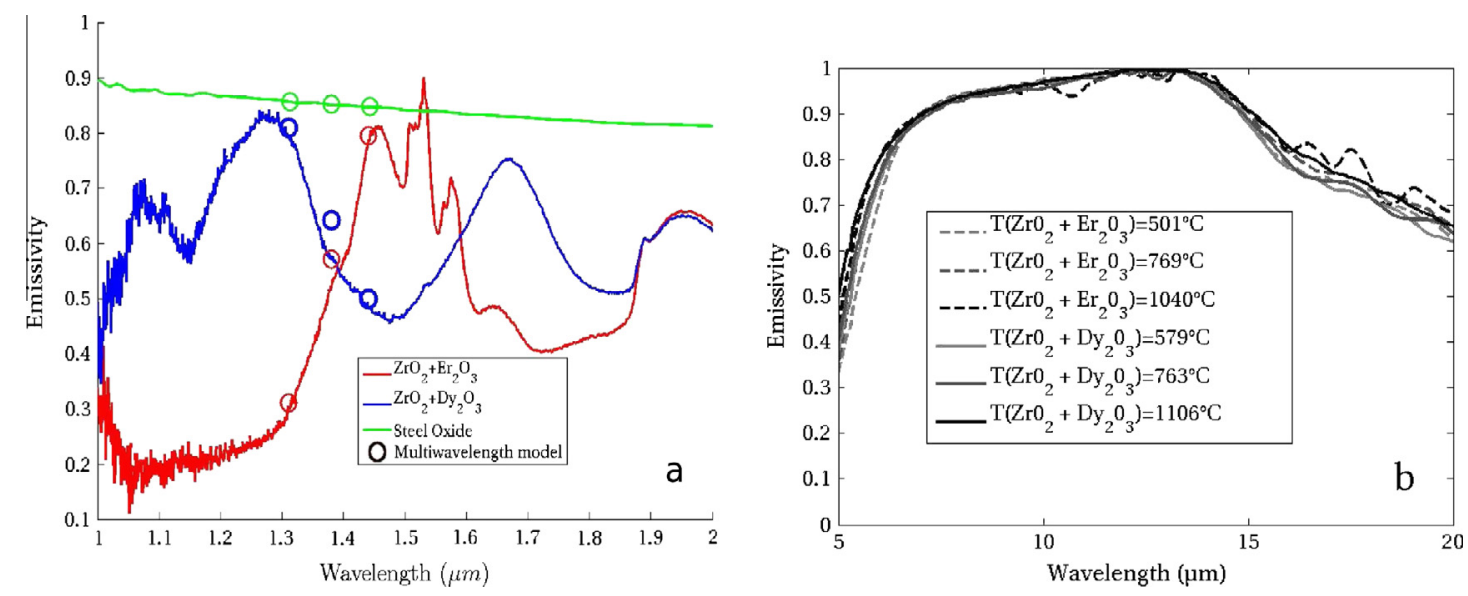

Fig. 2. Emissivity spectra of the scene in the near infrared (NIR) at room temperature for the three parts (a) and in the middle infrared (MIR) for several temperatures for the two dielectric parts (b). 
About the Steel Oxide part, the thermal conductivity is around $16 \mathrm{~W} \mathrm{~m}^{-1} \mathrm{~K}^{-1}$ as found in article [24]. However, the major influence quantity for the surface temperature is the thermal contact resistance, which is very high for this part. Indeed, it is not directly in contact with the heating plate, so the surface temperature will be lowered because of the presence of this air gap.

\subsection{Temperature error of passive methods}

From the previous radiative properties, theoretical temperature errors of conventional passive methods (monochromatic, bichromatic and multiwavelength thermography) are computed.

\subsubsection{Monochromatic thermography}

Principle. The temperature $T_{1 \lambda}$ provided by a monochromatic system is obtained using Eq. (1), expressing the signal $S_{0}$ following Planck's law. The error on temperature is then given Eq. (2).

$\frac{\Delta T_{1 \lambda}}{T_{1 \lambda}}=\frac{\lambda T_{1 \lambda}}{C_{2}} \frac{\Delta \varepsilon^{r}}{\varepsilon^{r}}$

Error incurred on our scene. (1) For the dielectric parts, as the emissivity varies a lot with wavelength (see Fig. 2), it is necessary to set the wavelength at a particular wavelength where emissivity is constant. As shown by Fig. 2, it would be wise to choose wavelength $12.8 \mu \mathrm{m}$ for a good accuracy in the whole thermal range. At this wavelength and at $1000^{\circ} \mathrm{C}$, a realistic emissivity error of $1 \%$ caused by the use of non-monochromatic filters leads to an absolute error on the temperature around $12^{\circ} \mathrm{C}$. (2) For the Steel Oxide part, emissivity is continuously changing because of the oxidation, so this method cannot be applied.

Application. This method set at Christiansen's wavelength will be used to provide a reference temperature for the dielectric parts.

\subsubsection{Bichromatic thermography}

Principle. Writing Eq. (1) for two wavelengths, and supposing that emissivity is constant for the wavelengths selected $\left(\varepsilon_{r}^{r}=1\right)$, we are free from the absolute knowledge of emissivity. The error involved by this approximation is written in Eq. (3):

$\frac{\Delta T_{2 \lambda}}{T_{2 \lambda}}=\frac{\Lambda T_{2 \lambda}}{C_{2}} \frac{\Delta \varepsilon_{r}^{r}}{\varepsilon_{r}^{r}} \quad$ with $\quad \varepsilon_{r}^{r}=\frac{\varepsilon_{1}^{r}}{\varepsilon_{2}^{r}} \quad$ and $\quad \Lambda=\frac{\lambda_{1} \lambda_{2}}{\lambda_{2}-\lambda_{1}}$

Error incurred on our scene. (1) For the dielectric parts, the emissivity varies a lot with wavelength, so this method cannot be applied. (2) For the Steel Oxide part, the behavior is almost as a gray body (the error on the emissivity ratio is $10 \%$ from $\lambda_{1}=1$ to $\lambda_{2}=2 \mu \mathrm{m}$ ), and the resulting error at $1000^{\circ} \mathrm{C}$ is $18^{\circ} \mathrm{C}$.

Application. This method will be used to provide a reference temperature for the Steel Oxide part.

\subsubsection{Multiwavelength thermography}

Principle. Writing Eq. (1) for $N$ wavelengths, and assuming that emissivity follows a model (see [9]) versus wavelength, it is possible to perform multiwavelength thermography. If the number of wavelengths (equations) is set to $N$, the degree of a polynomial model $\left(\varepsilon^{r}=\sum_{i=0}^{M} a_{i} \lambda^{i}\right)$ must be $M=N-2$. For our scene, and in the near infrared domain, the number of wavelengths will be limited to three in order to use a rather simple mathematical system for resolution and to avoid spectral band covering. With three wavelengths, the model must be linear, and the wavelengths where emissivity variations are the most linear are $1.31,1.38$ and $1.44 \mu \mathrm{m}$, as testified by Fig. 2 .

Error incurred on our scene. As there is no analytical form for the error, it is calculated numerically by solving Eq. (1) for three wavelengths and a temperature of $1000^{\circ} \mathrm{C}$. (1) For the dielectric parts, the error is -7 and $-9{ }^{\circ} \mathrm{C}$ respectively for the Erbium and the
Dysprosium oxide part. (2) The error is only $-19 \mathrm{~m}{ }^{\circ} \mathrm{C}$ for the Steel Oxide part.

Application. This method is very precise for this spectral domain. However, in practice, these wavelengths are very close and require the use of narrow band filters to avoid band covering. Such filters induce low signal detections and penalize middle-temperature measurement. Moreover, the choice of the wavelengths is very "material-dependent", so is possibly inefficient on other non-gray bodies. For these reasons, this method is not implemented.

Finally, these methods can be accurate but only for a specific part of the sample. Monochromatic thermography enables a reference temperature measurement on the dielectric part at $12.8 \mu \mathrm{m}$, where emissivity does not vary with temperature. Bichromatic thermography provides an accurate temperature for the Steel Oxide part, which is an almost gray body. Multiwavelength thermography is practically difficult to develop because of the closeness of the wavelengths where the model is valid, so would not be considered. In next part, an active method allowing a simultaneous measurement of temperature on the three parts will be presented.

\section{Thermoreflectometry method}

In this section, the active thermoreflectometry method is described.

\subsection{Principle of the method}

A recall of the theoretical basis of this technique is given with the main items: measurements, hypothesis and resolution. A complete description is given in [20].

\subsubsection{Indirect measurement of emissivity by reflectometry}

Thermoreflectometry proposes an indirect measurement of emissivity by reflectometry. For an opaque material and following Kirchhoff's laws, the directional spectral emissivity and the directional hemispherical spectral reflectivity $\rho^{r, \cap}$ obey the following equation:

$\varepsilon^{r}(\lambda, T)=1-\rho^{r, \cap}(\lambda, T)$

The directional hemispherical reflectivity is given by the integration in all directions $x$ of the bidirectional reflectivity $\rho^{r, x}$ which is carried out over the upward hemisphere $\cap$, as follows:

$\rho^{r, \cap}(\lambda, T)=\int_{\Omega_{x}=2 \pi} \rho^{r, x}(\lambda, T) \cos \left(\theta_{x}\right) d \Omega_{x}$

The first step of the method is to perform the measurement of the bidirectional reflectivity in a single direction $i$, called $\rho^{r, i}(\lambda, T)$. The directional hemispherical reflectivity is then expressed with respect to the measured bidirectional reflectivity as follows:

$\rho^{r, \cap}(\lambda, T)=\rho^{r, i}(\lambda, T) \int_{\Omega_{x}=2 \pi} f^{r, x, i}(\lambda, T) \cos \left(\theta_{x}\right) d \Omega_{x}$

where $f^{r, x, i}$ is the reflection indicator function, which represents normalized values of bidirectional reflectivities.

\subsubsection{Introduction of the diffusion factor}

The second step is to consider the previous integral as an unknown variable equal to the diffusion factor $\eta$ as follows:

$\eta^{r, i}(\lambda, T)=\int_{\Omega_{r}=2 \pi} f^{r, x, i}(\lambda, T) \cos \left(\theta_{x}\right) d \Omega_{x}$

For an opaque material, the diffusion factor is a shape factor, mainly geometrical, and it represents the volume of the bidirectional reflectivities normalized in the reference direction $i$. 
It characterizes the proportion of reflected radiation in the direction of measurement with regard to the reflected radiation scattered over the hole hemisphere.

According to Eqs. (4, 6 and 7) and thanks to Helmholtz's reciprocity theorem applied to bidirectional reflectivity $\rho^{r, i}(\lambda, T)$, the emissivity is then expressed as a function of the measured bidirectional reflectivity in a single direction $r$ and the unknown diffusion factor, as follows:

$\varepsilon^{r}(\lambda, T)=1-\rho^{i, r}(\lambda, T) \eta^{r, i}(\lambda, T)$

Eq. (8) shows that emissivity has a physical sense only if the diffusion factor is positive and less than $1 / \rho^{i, r}$.

\subsubsection{Main hypothesis and bichromatic system}

The third step consists in assuming that the diffusion factor is constant for two wavelengths $\eta^{r, i}\left(\lambda_{1}, T\right)=\eta^{r, i}\left(\lambda_{2}, T\right)$ and to re-write Eq. (1) for those two wavelengths and with Wien's approximation (valid for [0.9-1.7] $\mu \mathrm{m}$ spectral and [300-1000] ${ }^{\circ} \mathrm{C}$ thermal ranges, when $\lambda T \leqslant 3000 \mu \mathrm{m} \mathrm{K}$ ). Radiance temperature $T_{R}^{r}$ are measured by inverting the radiometric model chosen without considering emissivity value.

In a first approach, the hypothesis seems realistic because the diffusion of light by reflexion refers more to the roughness of the material than to its optical indexes. Hence, for two near wavelengths, the reflectivity shapes should be homothetics. Moreover, experimentally, this hypothesis has been verified for several materials and conditions [17].

The directional emissivity is replaced by Eq. (8) with the above assumption, and system (9) is obtained:

$$
\begin{aligned}
& \frac{1}{T_{2 \lambda, \rho}}=\frac{1}{T_{R}^{r}\left(\lambda_{1}\right)}+\frac{\lambda_{1}}{C_{2}} \ln \left(1-\eta^{r, i} \rho^{i, r}\left(\lambda_{1}, T\right)\right) \\
& \frac{1}{T_{2 \lambda, \rho}}=\frac{1}{T_{R}^{r}\left(\lambda_{2}\right)}+\frac{\lambda_{2}}{C_{2}} \ln \left(1-\eta^{r, i} \rho^{i, r}\left(\lambda_{2}, T\right)\right)
\end{aligned}
$$

Measuring radiance temperature and bidirectional reflectivity at two wavelengths, and assuming that shapes of reflectivity distribution are homothetic for both wavelengths, the system of Eq. (9) provides the true temperature $T_{2 \lambda, \rho}$ and the diffusion factor $\eta^{r, i}$.

\subsection{Temperature error}

As depicted by Eq. (10), the temperature error of thermoreflectometry is governed by two factors: the wavelength and the correctness of the hypothesis. Note that the error on radiance temperature and bidirectional reflectivities are considered as null to obtain this expression.

$\frac{\Delta T_{2 \lambda, \rho}}{T_{2 \lambda, \rho}}=2 \frac{\Lambda T_{2 \lambda, \rho}}{C_{2}} \frac{\Delta \eta}{\eta}$

About the wavelengths choice, $\Lambda$ is low if $\lambda_{1}$ are $\lambda_{2}$ are the farthest possible. About the correctness of the hypothesis, the diffusion factor is most likely constant if the wavelengths chosen are the closest, so a compromise must be reached.

To help us in that decision, some practical considerations must be taken into account. First of all, only wavelengths where laser emission exist have to be considered $(1.064,1.208,1.31,1.4$ and $1.55 \mu \mathrm{m}$ for the NIR spectral band). Secondly, we will try to have detection for rather-low temperatures, so we would not favor $1.064 \mu \mathrm{m}$ for the first wavelength. Thirdly, the wavelengths chosen must not be too close in order to avoid peak recovering when using filters.

To conclude, wavelengths 1.31 and $1.55 \mu \mathrm{m}$ provide a good compromise for a thermoreflectometer accurate on an unknown material.

\subsection{Apparatus}

The thermoreflectometer is a simple packaged instrument, located at $1 \mathrm{~m}$ from the heated scene and comprised of: (1) A near infrared camera and a near infrared lens. The possibility of adjusting the camera integration time related to the aperture of the lens opens a very wide temperature range from 300 to $1000^{\circ} \mathrm{C}$. The defects (non-uniform pixels, radiometric errors, chromatic aberrations) of the images have been characterized in $[19,18]$. Background subtraction is applied to each of the raw images, and corrects the majority of the defects. (2) The optical source is based on laser diodes. Only lasers, with their high spectral density power, could produce a beam powerful enough to be detected against thermal emissions. Moreover, the source has to illuminate a larger surface, so a stage of collimation and enlargement has been installed. This way we obtained a $5 \mathrm{~cm}$ diameter bichromatic beam at two near infrared wavelengths: 1.31 and $1.55 \mu \mathrm{m}$. The basic sources are continuous $20 \mathrm{~mW}$ laser diodes, which induces a satisfactory signal-to-noise ratio up to $1000^{\circ} \mathrm{C}$. (3) A motorized filter wheel provides the spectral selection of the camera at wavelengths 1.31 and $1.55 \mu \mathrm{m}$. (4) All the commands and treatments are managed by a specific software developed within the framework of this project.

To sum up, thermoreflectometry brings out several advantages: firstly, compared to monochromatic thermography, it does not require apriori knowledge of emissivity, because a measurement is performed. Secondly, compared to bichromatic thermography, and even if the theoretical error shows a factor 2 in the expression (see Eq.(10)), the hypothesis on the diffusion factor is less restrictive than the one on emissivity. Indeed, the diffusion factor is mostly a function of the roughness of the material, whereas emissivity is a function of both optical indexes and roughness of the material. Thirdly, its performances are less "material-dependent" than multiwavelength thermography, for which the wavelengths must be optimized for each material (domain where the model fits real variations).

\section{Experimental results}

In this section, the heating plate is set to a temperature of $850^{\circ} \mathrm{C}$, which is its maximum temperature. As testified by Eq. (10), these conditions should induce a maximum error on temperature. The thermoreflectometer visualizes the scene following the outer circle presented in Fig. 1. The entire determination of true temperature is given in this section, with the preliminary measurements of radiance temperatures and bidirectional reflectivities.

\subsection{Reference temperature}

To prove the accuracy of the results obtained, we need a reference method to simultaneously measure temperature with the thermoreflectometer.

- For the dielectric part (isolating and porous), the use of thermocouples is very difficult and poses the problem of thermal bridges. Referring to part 2, monochromatic thermography is performed with a $12.8 \mu \mathrm{m}$ filter mounted on a MIR microbolometer camera. The average temperature obtained, is 679 and $659^{\circ} \mathrm{C}$, respectively for the Erbium and the Dysprosium oxide part: this gradient of $20^{\circ} \mathrm{C}$ can be attributed to the thermal conductivity difference characterized in Section 2.

- For the Steel Oxide part, two thermocouples have been welded on the coldest part. They measured temperatures of 608 and $611^{\circ} \mathrm{C}$. However, these are punctual measurements, and represent the minimum reference temperature because they are 
located on the coldest part of the region of interest. On the contrary, bichromatic thermography displays a mean temperature of $628^{\circ} \mathrm{C}$ in the region of interest.

\subsection{Radiance temperatures}

The radiance temperature field at $1.31 \mu \mathrm{m}$ is given in Fig. 3, as an image and a histogram. The results for the other wavelength $1.55 \mu \mathrm{m}$ are presented in Table 1 , in the form of spatial means and standard deviations.

For the dielectric part, one can notice the very good separation of the two parts, which testifies to the difference of emissivity, and, to a lesser degree, to the thermal conductivity, between the two parts. Hence, the two peaks are well separated in the histogram. For the Erbium oxide part, the spatial mean increases with wavelength, from $624^{\circ} \mathrm{C}$ at $1.31 \mu \mathrm{m}$ to $665^{\circ} \mathrm{C}$ at $1.55 \mu \mathrm{m}$, in conformity with the emissivity variation. Each area is relatively homogeneous, with a standard deviation of only $4^{\circ} \mathrm{C}$, which is probably due to the better mechanical contact of the upper and the bottom side of the sample, both of which are hotter than the center. For the Dysprosium part, the spectral variation is inverted, as is emissivity. The spatially averaged radiance temperature is equal to $654^{\circ} \mathrm{C}$ at $1.31 \mu \mathrm{m}$ and $635^{\circ} \mathrm{C}$ at $1.55 \mu \mathrm{m}$.

For the metallic part, the radiance temperature is low due to the high contact thermal resistance between the flange and the heating plate. The radiance temperatures for both wavelengths are very close, which corresponds to a gray body behavior.

\subsection{Bidirectional reflectivity measurement}

The bidirectional reflectivity field at $1.31 \mu \mathrm{m}$ is given in Fig. 4, as an image and a histogram. See Table 1 for the other wavelength. About the dielectric part, the left side of the sample is now brighter than the right side, contrary to the previous image of radiance temperatures. This is due to the fact that emissivity goes down as reflectivity goes down. At $1.31 \mu \mathrm{m}$, the Erbium oxide part reflects more than the Dysprosium oxide part, which can be linked to the emissivity spectrum. Here also, the two peaks are well separated.

For the metallic part, bidirectional reflectivity is low because of the oxidized surface, and slightly increases with wavelength, in conformity with the emissivity spectrum.

Then, one can notice that the bidirectional reflectivity field uniformity is worse than for radiance temperature. This phenomenon is explained by the fact that bidirectional reflectivity is not only an intrinsic physical property of the materials, but also depends on the configuration of the detector. More precisely, it depends on the angle between the pixel and the surface normal. As each pixel involves different angles, bidirectional reflectivity also varies.
Table 1

Results of spatial means and standard deviations obtained by thermoreflectometry on the three parts of the scene.

\begin{tabular}{llllll}
\hline & & $\bar{x}\left(\lambda_{1}\right)$ & $\sigma_{x}\left(\lambda_{1}\right)$ & $\bar{x}\left(\lambda_{2}\right)$ & $\sigma_{x}\left(\lambda_{2}\right)$ \\
\hline $\mathrm{ZrO}_{2}-\mathrm{Er}_{2} \mathrm{O}_{3}$ & $x=\rho^{i, r}\left(\mathrm{sr}^{-1}\right)$ & $3.13 .10^{-1}$ & $3.0 .10^{-2}$ & $1.13 .10^{-1}$ & $1.30 .10^{-2}$ \\
& $x=T_{R}^{r}\left({ }^{\circ} \mathrm{C}\right)$ & 624 & 4 & 665 & 7 \\
& $x=T_{r e f}\left({ }^{\circ} \mathrm{C}\right)$ & 679 & 11 & - & \\
& $x=T_{2 \lambda, \rho}\left({ }^{\circ} \mathrm{C}\right)$ & 688 & 7 & - & \\
& $x=\eta^{r, i}(\mathrm{sr})$ & 1.80 & 0.22 & - & \\
& $x=\varepsilon^{r}$ & 0.443 & 0.025 & 0.796 & 0.036 \\
$\mathrm{ZrO}_{2}-\mathrm{Dy}_{2} \mathrm{O}_{3}$ & $x=\rho^{i, r}\left(s r^{-1}\right)$ & $8.40 .10^{-2}$ & $1.00 .10^{-2}$ & $1.64 .10^{-1}$ & $2.4 .10^{-2}$ \\
& $x=T_{R}^{r}\left({ }^{\circ} \mathrm{C}\right)$ & 654 & 6 & 635 & 5 \\
& $x=T_{r e f}\left({ }^{\circ} \mathrm{C}\right)$ & 659 & 10 & - & \\
& $x=T_{2 \lambda, \rho}\left({ }^{\circ} \mathrm{C}\right)$ & 667 & 7 & - & \\
& $x=\eta^{r, i}(\mathrm{sr})$ & 1.84 & 0.38 & - & 0.033 \\
$\mathrm{Steel} \mathrm{Oxide}=\varepsilon^{r}$ & 0.846 & 0.032 & 0.705 & 0.031 \\
& $x=\rho^{i, r}(\mathrm{sr}-1)$ & 0.053 & 0.007 & 0.067 & 3 \\
& $x=T_{R}^{r}\left({ }^{\circ} \mathrm{C}\right)$ & 618 & 2 & 617 & 3 \\
& $x=T_{r e f}\left({ }^{\circ} \mathrm{C}\right)$ & 628 & 7 & - & \\
& $x=T_{2 \lambda, \rho}\left({ }^{\circ} \mathrm{C}\right)$ & 622 & 14 & - & \\
& $x=\eta^{r, i}(\mathrm{sr})$ & 0.792 & 3.072 & - & 0.134 \\
\hline$x$ & $=\varepsilon^{r}$ & 0.959 & 0.155 & 0.959 & \\
\hline
\end{tabular}

\subsection{True temperature retrieving}

With the radiance temperature and the bidirectional reflectivity fields at two wavelengths, it is possible to determine the true temperature field using system (9). It is displayed in Fig. 5 and the mean values are presented in Table 1.

Analyzing the image on the dielectric part, the sample looks less heterogeneous than for the previous measurements. Indeed, the two spatial means of temperatures $\left(688^{\circ} \mathrm{C}\right.$ for $\mathrm{Er}_{2} \mathrm{O}_{3}$ and $667{ }^{\circ} \mathrm{C}$ for $\mathrm{Dy}_{2} \mathrm{O}_{3}$ ) are separated by $21^{\circ} \mathrm{C}$ and parts of the two peaks are superimposed. The differences with the reference temperatures are only 9 and $8{ }^{\circ} \mathrm{C}$ respectively for the Erbium and the Dysprosium part. Moreover, the measured temperature difference between the two parts $\left(21^{\circ} \mathrm{C}\right)$, is close to that of the reference $\left(20^{\circ} \mathrm{C}\right)$.

For the metallic part, the spatially averaged temperature retrieved is low $\left(622^{\circ} \mathrm{C}\right)$ and close to the radiance temperature one, due to the high emissivity value. The difference with the reference temperature obtained by bichromatic thermography is only $6{ }^{\circ} \mathrm{C}$. The high dispersion of temperature on the area of interest is explained by the distribution of temperature on the flange. The dielectric sample heats the flange at the contact area, and the temperature decreases as we get distant from the sample.

The important conclusion is that the value retrieved on this sample is very close from the reference temperature, this allows
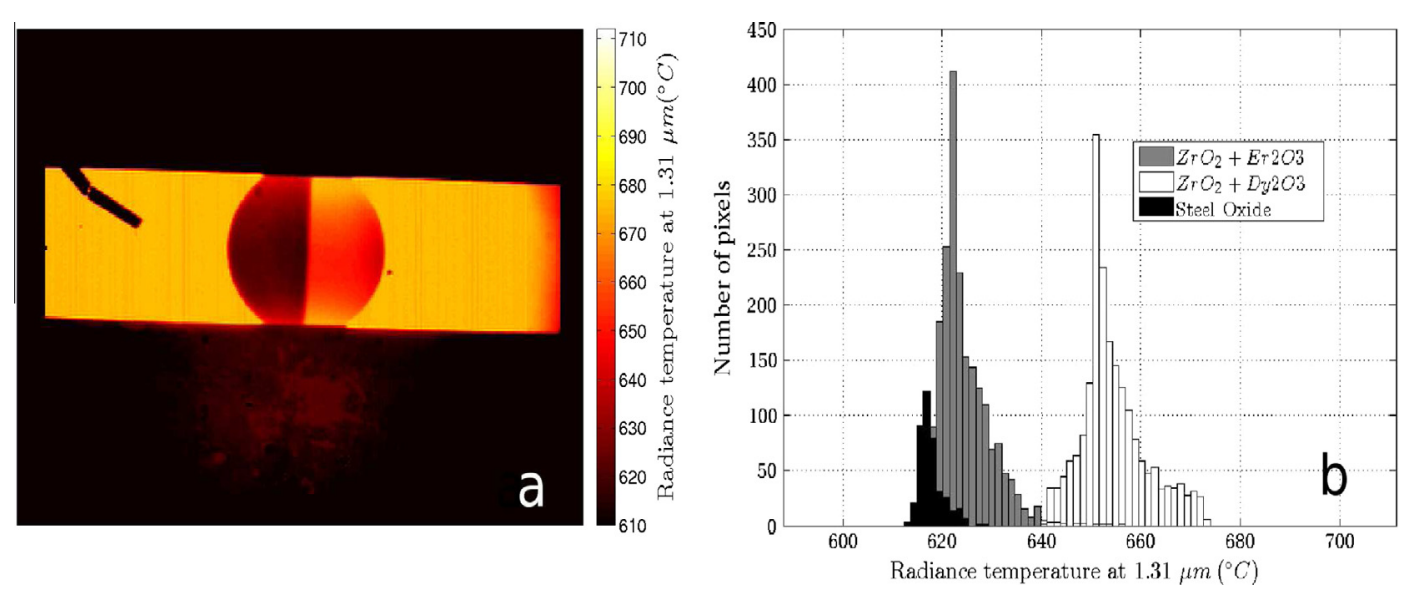

Fig. 3. Radiance temperature field at $1.31 \mu \mathrm{m}$ as an image (a) and a histogram (b). 

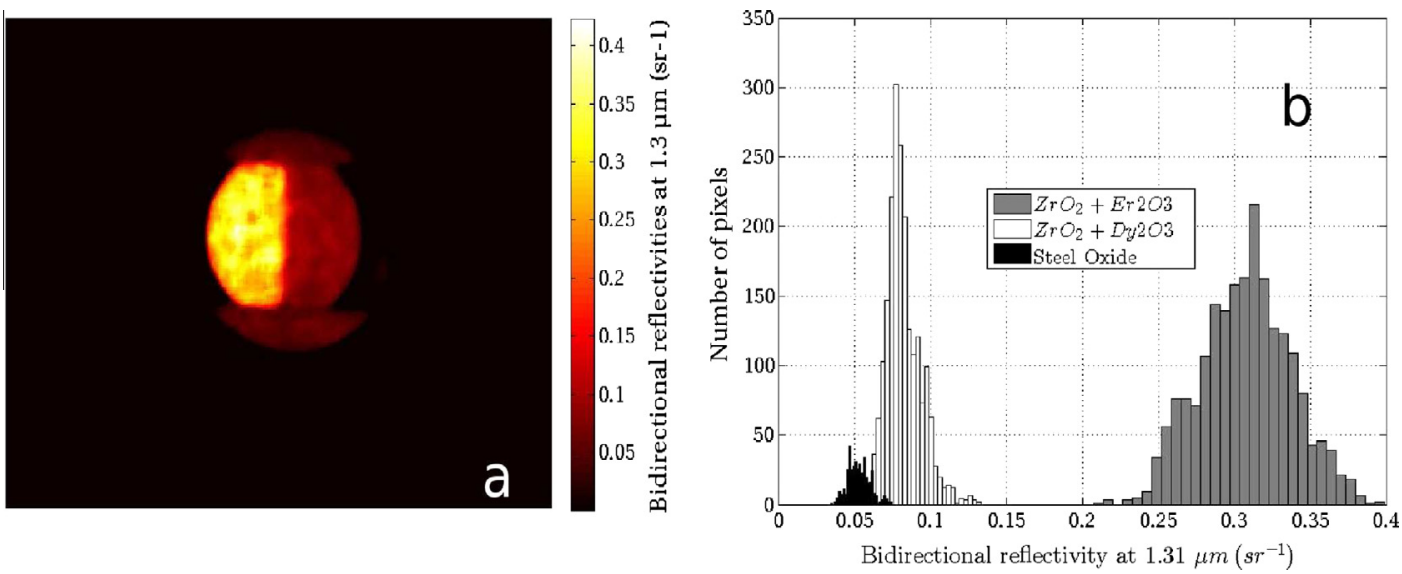

Fig. 4. Bidirectional reflectivity field at $1.31 \mu \mathrm{m}$ as an image (a) and a histogram (b).
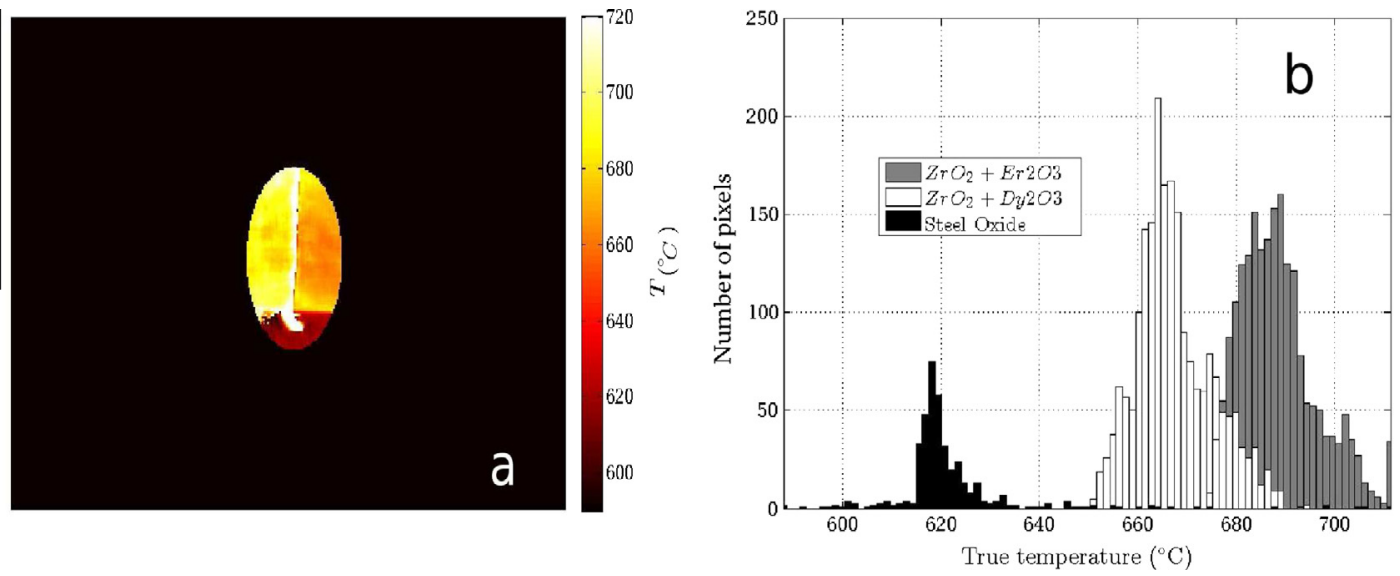

Fig. 5. True temperature field as an image (a) and a histogram (b).
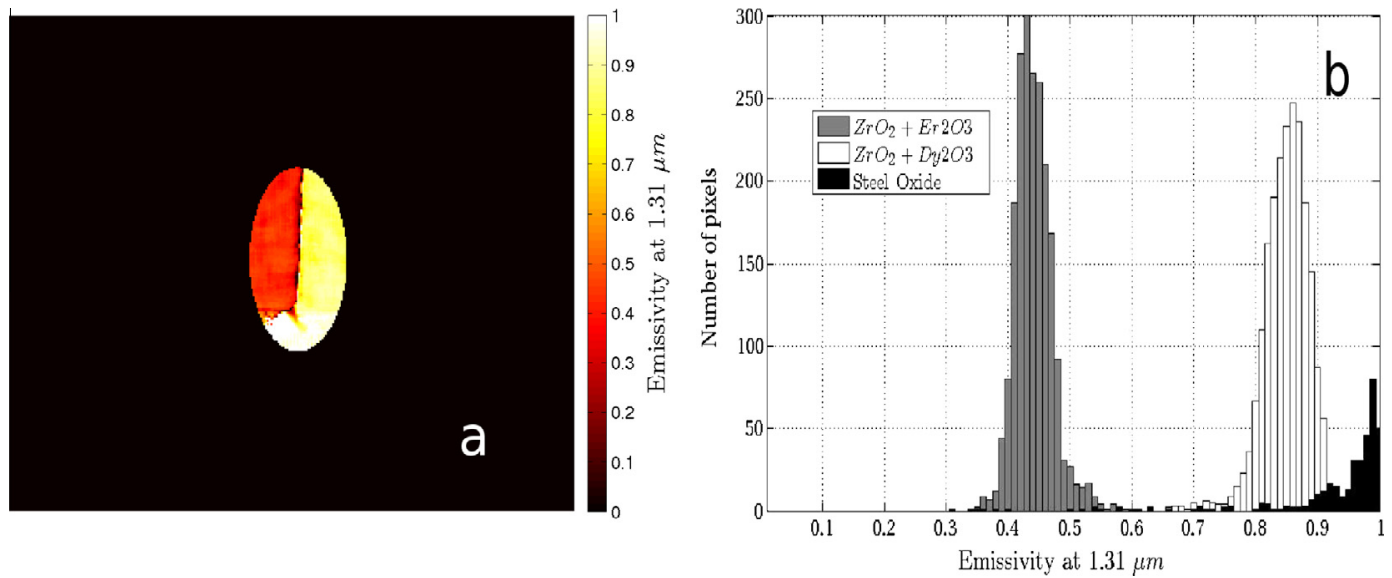

Fig. 6. Emissivity field at $1.31 \mu \mathrm{m}$ as an image (a) and a histogram (b).

us to state that thermoreflectometry is a quantitative temperature field measurement method.

The diffusion factor is also calculated, and its mean value is presented in Table 1. As noticed for the characterization part, the diffusion factor is the same for the two dielectric parts, which supposes that the type of reflection is the same for the two parts and that the hypothesis of the method is respected for this sample. For the metallic sample, its value is lower because oxidized metals diffuses less light than a high-roughness dielectric. These results have then a physical meaning and reinforce the physical consistency of the method.

\subsection{Emissivity fields}

With the bidirectional reflectivity and the diffusion factor field, it is possible to calculate the emissivity field. Fig. 6 shows the emissivity field at $1.31 \mu \mathrm{m}$ and the mean values for the other wavelengths are presented in Table 1. 
About the dielectric part, the two parts are very distinct, as testified by the peak separation of the histogram. This corresponds to the strong non-gray behavior of this sample, enhanced by the high emissivity difference for the two dopants for the wavelength $1.31 \mu \mathrm{m}$. The metallic part exhibit the highest emissivity, which almost does not vary with wavelength (gray body).

However, the emissivities are a bit different than the one characterized at room temperature in Fig. 2. This phenomenon can be explained by the effect of temperature on emissivity, which has been quantified in the article [5], and is more important for the part of the spectrum involving low emissivities. On the metallic part, it is explained by the continuous oxidation of the surface.

\subsection{Results summary}

All the results are summarized in Table 1, in the form of spatial means and standard deviations.

These results show the high consistency of thermoreflectometry. On a scene considered to be a very difficult case for non-contact measurements because emissivity varies with the pixel considered and the temperature, our method has retrieved the surface temperature field accurately and on-line. Moreover, the emissivity field of the sample at high temperature has also been determined, which is a crucial criteria for evaluating the surface of the sample, and offers interesting prospects for the in situ control of the surface state.

\section{Conclusion}

The main objective of this paper was to emphasize the temperature error of thermoreflectometry, a new method of temperature field measurement, on a multi-materials scene divided in three materials: two dielectrics composed of rare earth oxides dopants and one metal oxide.

The multi-materials scene represents a difficult case for noncontact temperature measurement and exhibits an emissivity and temperature gradient over the surface. The experimental measurement of radiative and thermal properties demonstrates that the dielectric material is a non-gray body, and emissivities of the two parts vary in opposite ways versus wavelength. The metal oxide material is an almost gray body. The calculation of temperature error of conventional passive methods emphasizes the fact that: (1) Monochromatic thermography at Christiansen's wavelength can provide a reference temperature for the dielectric part, but is not suitable to the metal oxide one. (2) Bichromatic thermography performs a low temperature error on the metal oxide part, but a high error on the dielectric one. (3) Multiwavelength thermography, with a polynomial model expressed at first order, is accurate if the spectral band chosen is very short, which restricts a lot the method due to practical reasons (filters' bandwidth, signal's amplitude).

The suggested method which performs a low temperature error for all materials is based on the measurement, at two wavelengths, of the radiance temperatures in a passive step, and of bidirectional reflectivity in an active step. The knowledge of these two quantities enables the true temperature to be determined in dynamical conditions. The simple apparatus was presented, and the choice of the influence quantities (hypothesis, wavelengths) was discussed. The wavelengths 1.31 and $1.55 \mu \mathrm{m}$ are well adapted to this scene.

Thirdly, the multi-materials scene was heated to a temperature of $850^{\circ} \mathrm{C}$ on its back side, and the temperature measurement was performed on the front side. Despite the strong variations of radi- ative properties, the true temperature field retrieved is accurate. In each case the temperature was compared to that of a reference measurement. Moreover, the emissivities calculated by thermoreflectometry were very distinct from material to material. This testifies to the overall consistency of the method.

To sum up, this prototype is able to perform true temperature measurement on very non-gray scenes, with a possible variation of emissivity with space, wavelength and time. The diffusion factor calculation enables indirectly the in situ control of the surface state, and could detect some physical phenomena such as oxidation, delamination or phase transformations.

After the investigation of the influence of emissivity on temperature (this paper) and of convection [25], another influence quantity is now being treated in our laboratory: the shape of the object, which involves the under- or over-estimation of the temperature caused by multi-reflections. Following this approach, the fusion of thermoreflectometry with shape reconstruction performed in the near infrared domain is now in progress.

\section{Acknowledgements}

The team would like to thank the CEMHTI laboratory for the mid-infrared thermal emissivity characterization.

This work was carried out in the context of the R3T (Real Time True Temperature) project, supported by the French National Research Agency.

\section{References}

[1] C. Lempereur, R. Andral, J.Y. Prudhomme, Meas. Sci. Technol. 19 (2008) 105501.

[2] R. Reichle, P. Andrew, C. Balorin, B. Brichard, S. Carpentier, Y. Corre, M. Davi, R. Daviot, C. Desgranges, J. Gardarein, E. Gauthier, D. Guilhem, S. Gicquel, A. Herrmann, D. Hernandez, M. Jouve, C.L. Niliot, T. Loarer, A. Martin, J. Martins, J.B. Migozzi, J. Patterlini, C. Pocheau, F. Rigollet, H. Roche, J. Travere, J. Nucl. Mater. 390-391 (2009) 1081-1085. Proceedings of the 18th International Conference on Plasma-Surface Interactions in Controlled Fusion Device.

[3] M. Balat-Pichelin, D. Hernandez, G. Olalde, B. Rivoire, J. Robert, American Society of Mechanical Engineers, New York, NY, ETATS-UNIS, vol. 124, 2002, pp. 215-222.

[4] V.S. Dozhdikov, V.A. Petrov, High Temp. High Press. 27-28 (1995) 403-410.

[5] O. Rozenbaum, D.D.S. Meneses, Y. Auger, S. Chermanne, P. Echegut, Rev. Sci. Instrum. 70 (10) (1999) 4020-4025.

[6] F. Meriaudeau, Image Vision Comput. 25 (2007) 1124-1133.

[7] T. Pierre, B. Remy, A. Degiovanni, J. Appl. Phys. 103 (2008) 034904.

[8] J. Thevenet, M. Siroux, B. Desmet, Appl. Therm. Eng. 30 (2010) 1359-4311.

[9] T. Duvaut, Infrared Phys. Technol. 51 (2008) 292-299.

[10] D.L. Balageas, High Temp. - High Press. 21 (1989) 85-96. ONERA, TP No. 1989155, 1989, 13 p. 21 (1989) 85-96.

[11] K. Chatterjee, S. Tuli, S.G. Pickering, D.P. Almond, NDT amp; E Int. 44 (2011) 655-667.

[12] J. Renshaw, J.C. Chen, S.D. Holland, R.B. Thompson, NDT amp; E Int. 44 (2011) 736-739.

[13] E. Kuhn, E. Valot, P. Herve, Compos. Struct. 94 (2012) 1155-1164.

[14] B. Weekes, D.P. Almond, P. Cawley, T. Barden, NDT amp; E Int. 49 (2012) 4756.

[15] T. Salikhov, V. Kan, Appl. Solar Energy 39 (2006) 66-76.

[16] H.R. Tschudi, M. Schubnell, Rev. Sci. Instrum. 70 (1999) 2719-2727.

[17] D. Hernandez, Rev. Sci. Instrum. 76 (2005). pp. 024904-024904-7.

[18] R. Gilblas, Measurement of True Temperature Field by Near Infrared Thermography, Ph.D. thesis, 2012.

[19] T. Sentenac, R. Gilblas, Metrologia 50 (2013) 208-218.

[20] T. Sentenac, R. Gilblas, D. Hernandez, Y. Le Maoult, Rev. Sci. Instrum. 83 (2012). pp. 124902-124902-14.

[21] L. del Campo, D. De Sousa Meneses, A. Blin, B. Rousseau, E. Vron, M. BalatPichelin, P. Echegut, J. Am. Ceram. Soc. 94 (2011) 1859-1864.

[22] S.E. Gustafsson, Rev. Sci. Instrum. 62 (1991) 797-804.

[23] B. Jang, J. Sun, S. Kim, Y. Oh, H. Kim, Surface Coat. Technol. 207 (2012) 177181.

[24] H. Boyer, T. Gall, Metals Handbook, desk ed., 1985.

[25] A. Delmas, Y. Le Maoult, J.-M. Buchlin, T. Sentenac, J.-J. Orteu, Exp. Fluids 54 (2013) 1-16. 